\title{
Estimulación temprana y desarrollo psicomotor en niños de 4 a 5 años.
}

\section{Early stimulation and psychomotor development in children 4 to 5 years.}

Helder Marcell Barrera Erreyes. ${ }^{1}$, Ana Rebeca Flor Castelo. ${ }^{2}$ \& Fernando Alfredo Flor Tapia $^{3}$

Recibido: 12-09-2017 / Revisado: 08-11-2017 Aceptado: 05-12-2017/ Publicado: 01-01-2018

\begin{abstract}
.
DOI: $\underline{\text { https://doi.org/10.33262/cienciadigital.v2i1.5 }}$

The early stimulation study considers a variety of actions, which have as a priority the development of children in their first years of life, and, likewise it provides an extensive range of activities, which affect in building of its live daily in society, which is evidenced in behavior. The human being is eminently social; therefore, it develops the interpersonal relationships with others where it acquires affective bonds as friendship, respect, attachment, among others. This study aims to show that if it is given at children early stimulation of quality, it will be achieved in the future optimal psychomotor development, which will be reflected in their physical and intellectual abilities, ensuring their proper development and relationship with society. This research was hypothetical - deductive, because from the problem statement was perceived by observing the reality of it, it was based on a theoretical framework, the formulation of the hypothesis, then conclusions and recommendations were developed. The girls and boys of Initial Education Center "Eloy Alfaro" were the study subjects, who were observed and then it was applied the early stimulation techniques and procedures, which will contribute to psychomotor development. Based on data analysis was obtained very important parameters, which allow more understanding of how early stimulation is a key factor in the social skills development in children in preschool age. It is concluded that the analysis and proper implementation of early stimulation techniques through motor games, application graphic-plastic techniques and pedagogical techniques (cognitive) allow the correct psychomotor development of the boys and girls from four to five years old.
\end{abstract}

\footnotetext{
1 Universidad Técnica de Ambato, Pontificia Universidad Católica del Ecuador, Ambato, Ecuador, hm.barrera@uta.edu.ec

${ }^{2}$ Universidad Nacional de Chimborazo, Chimborazo- Ecuador, aflor@unach.edu.ec

${ }^{3}$ Pontificia Universidad Católica del Ecuador, Ambato- Ecuador, fflor@pucesa.edu.ec
} 
Keywords: Early Stimulation, Psychomotor Development, Childhood, Human Development, Children.

\section{Resumen.}

La estimulación temprana considera variedad de acciones cuya prioridad es el desarrollo del niño en sus primeros años de vida y brinda una extensa gama de actividades que inciden en su desarrollo psicomotor. El ser humano es eminentemente social, por lo tanto, resuelve sus necesidades básicas en relación con los demás y es donde adquiere vínculos afectivos como amistad, respeto, apego, entre otros. El presente estudio demuestra que si otorgamos estimulación temprana de calidad obtendremos un óptimo desarrollo psicomotriz reflejado en habilidades físicas e intelectuales. Esta investigación tiene carácter hipotético - deductivo, porque a partir del enunciado del problema y a través de la observación se percibió la realidad del mismo, se fundamentó en un marco teórico y planteamiento de la hipótesis para posteriormente elaborar conclusiones. Sujetos de estudio fueron las niñas y niños del Centro de Educación Inicial "Eloy Alfaro" de la provincia de Chimborazo y a partir de la observación se procedió a la aplicación de técnicas y procedimientos de estimulación temprana que contribuyan al desarrollo psicomotor. Del análisis de datos se obtuvieron parámetros importantes que ratificaron a la estimulación temprana como factor fundamental en el desarrollo de habilidades sociales en los niños en edad pre escolar, concluyendo que el análisis y aplicación adecuada de técnicas de estimulación temprana a través de juegos motores, aplicación de técnicas grafo plásticas y técnicas pedagógicas permiten el correcto desarrollo psicomotriz de los infantes.

Palabras Claves: Estimulación temprana, desarrollo psicomotor, infancia, desarrollo humano, niños.

\section{Introducción.}

El estudio del desarrollo humano se ha convertido en una de las principales fuentes de conocimiento que ha posibilitado la detección oportuna de situaciones personales adversas, así como la construcción de herramientas importantes para profesionales de diferentes áreas, puesto que el entendimiento de determinados factores que influyen en el proceso de cambio permite establecer estrategias para prevenirlos, modificarlos e intervenir sobre ellos de manera oportuna.

La educación del individuo desde los primeros años debe ir enfocada a la adquisición y desarrollo de sus capacidades, de modo que en el futuro puedan actuar con madurez, responsabilidad, respeto y propagación adecuada de su autonomía personal para adquirir conocimientos y destrezas que les permitan integrarse al máximo en el grupo social al que pertenecen, todo cuanto el niño hace o deja de hacer durante sus primeros años de vida influye 
en su ulterior perfeccionamiento intelectual, conforme madura es preciso que participe activamente en nuevas experiencias que contribuyan a establecer las bases fundamentales de los aprendizajes.

Durante la infancia, las capacidades motoras y sensorio perceptuales se desarrollan de forma recíproca, paralela al proceso mismo de maduración y mielinización del sistema nervioso, cuyas estructuras se encuentran organizando, diferenciando y especializando sus funciones, que cada vez serán menos motoras y más reguladas por el lenguaje. En términos de los procesos evolutivos, el desarrollo físico es considerado durante los primeros años de vida como la base sobre la que se establece el avance psicológico, aunque éste sea bastante independiente de las características físicas (Deval, 2006); de este modo, el proceso motor ocupa un lugar intermedio entre el desarrollo físico y el psicológico, al depender no sólo del impulso de los músculos y nervios relacionados sino también de capacidades sensorio perceptivas.

Varios son los compendios analizados en el ámbito local e internacional, una vez revisados los archivos de la biblioteca de la Facultad de Ciencias de la Educación, Humanas y Tecnologías de la Universidad Nacional de Chimborazo, se encontraron temas relacionados con la presente investigación, mismos que contribuyen como fuente de consulta para sustentar el problema planteado.

"La estimulación temprana en el desarrollo psicomotriz de los niños y niñas de 3 a 4 años con discapacidad de la Unidad Educativa especializada "Carlos Garbay" de la ciudad de Riobamba, Provincia de Chimborazo, Año Lectivo 2013 - 2014”.

Este tipo de investigaciones evidencian que el desarrollo psicomotor es un proceso continuo a través del cual el niño adquiriere habilidades gradualmente complejas que le permiten interactuar con personas, objetos y situaciones de su entorno en diversas formas. Incluye las funciones de la inteligencia y el aprendizaje, por medio de las cuales entiende y organiza su mundo, amplía la capacidad de comprender, hablar su idioma, desplazarse, manipular objetos, relacionarse con los demás y la expresión de sus emociones y sentimientos.

El objetivo principal de este trabajo es determinar la relación entre la estimulación temprana y el desarrollo psicomotor de los niños y niñas de cuatro a cinco años del Centro de Educación Inicial "Eloy Alfaro" de la ciudad de Riobamba, provincia de Chimborazo, tema que obtuvo interés previa la aplicación de la ficha de observación a los estudiantes y la encuesta a las maestras del mencionado Centro de Educación Inicial, para posteriormente sugerir actividades, juegos y tareas que fortalezcan el esquema corporal, salud mental, emocional y motriz de los niños y niñas. 


\section{Desarrollo.}

La Organización Mundial de la Salud refiere que el 60\% de los niños menores de 6 años no se encuentran estimulados, lo cual puede condicionar un retraso en cualquiera de sus tres esferas, describiendo además que la falta de estimulación temprana se da más en áreas rurales debido a la educación de los padres.

La Estimulación Temprana es el conjunto de acciones dirigidas a desarrollar las capacidades físicas, mentales y sociales del niño-a prevenir el retardo psicomotor, las alteraciones motoras, los déficit sensoriales, las discapacidades intelectuales, los trastornos del lenguaje y, sobre todo, a lograr la inserción de estos niños en su medio, sustituyendo la carga de una vida inútil por la alegría de una existencia útil y transformando los sentimientos de agresividad, indiferencia o rechazo en solidaridad, colaboración y esperanza,

"La Estimulación Temprana es una actividad basada principalmente en las neurociencias, en la pedagogía y en la psicología cognitiva, que favorece el desarrollo integral del niño"(Cultural, S.A., 2005).

El objetivo de la Estimulación Temprana no es acelerar el desarrollo, forzando al niño a lograr metas para las que no está preparado o para cumplir, si no el reconocer y motivar el potencial de cada niño en particular y presentarle retos y actividades adecuadas que fortalezcan su desarrollo físico y psicológico.

Desde el punto de vista humano, se entenderá como un proceso gradual y continuo de modificaciones relacionadas con la cantidad y calidad de las conductas que una persona va presentando durante su vida. Comienza en el momento mismo de la concepción, al dar lugar al crecimiento de un ser vivo en el seno materno; su evolución posterior al nacimiento y la suma de situaciones que tiene que ver con la herencia, la genética, es decir, lo propiamente orgánico y la influencia de los elementos ambientales como la alimentación, la salud, estímulos, afecto, educación y la interacción con los grupos circundantes como la familia, la comunidad y sociedad, mismos que influyen en el desenvolvimiento físico y psicológico del ser. (Unidas, 1990).

En el caso de los niños en edad preescolar, se hace referencia a los términos de crecimiento y desarrollo como procesos. El crecimiento es el aumento de peso y de las dimensiones de todo el organismo y de las partes que lo conforman, las cuales se miden por kilogramos y centímetros, así pues, el aumento regular de peso y talla es el indicador más confiable, para afirmar, que el niño goza de buen estado de salud física, lo que se invidencia cuando los indicadores antropométricos se mantienen en los estándares de clasificación normal respecto al peso, edad y talla de los infantes (Hernández y Rodríguez, 2004). 
Para medir el desarrollo se debe considerar la bio diferenciación y madurez de las células que conlleva la adquisición de destrezas y habilidades en varias etapas de la vida. Entonces entendiendo el desarrollo como un proceso, éste se caracteriza por ser continuo: porque se inicia desde el nacimiento y está presente a lo largo de toda una vida con la existencia de cambios y funciones que no se detienen, sino que van orientándose a niveles de mayor complejidad (Solis Torres, 2006).

Es importante saber que el desarrollo psicomotor es cefalocaudal: primero se adquieren las funciones motoras de la cabeza (como el sostén cefálico) y luego las de las piernas y próximodistal: desde el centro del cuerpo hacia fuera (de la columna vertebral hacia las manos). Además, sabemos que primero aparecerán movimientos reflejos o involuntarios que, con el juego y el hacer, se convertirán en movimientos voluntarios.

Además, se puede decir que el desarrollo psicomotor es secuencial (se adquieren capacidades siguiendo un orden, la primera va a ayudar a conseguir la siguiente), progresivo (las adquisiciones son cada vez más complejas) y coordinado (intervienen distintas capacidades).

La Estimulación Temprana es importante por tener un sustento científico para su aplicación en el proceso de desarrollo en las diferentes etapas en niños y niñas, es acogida como parte de la formación profesional a educadores parvularios, que con conocimientos relacionados con la realidad ponen de manifiesto sus capacidades. También se debe señalar que la Estimulación Temprana facilita el desarrollo de las habilidades y destrezas de todos los movimientos y reacciones naturales, para potenciar de manera organizada y progresiva en cada una de las etapas de desarrollo de niños y niñas, varios autores coinciden en que es más recomendable estimular a los niños que tengan edad cronológica de 0 a 6 años porque es en ese período donde se aprovechan de mejor manera los procesos de aprendizaje.

Hay una mielinización del Sistema Nervioso Central que hará posible establecer anormalidades del desarrollo (Indemnidad Cerebral). Muchos padres dedican un mayor tiempo a sus hijos en los primeros años de vida y entonces se hace más realista y fácil incluirlos en los programas de estimulación (valor afectivo). Es la época en que los padres requieren de un apoyo más cercano de aquellas personas que comprenden el problema de manera positiva y que le permitan entender mejor los problemas que están presentando sus hijos. El programa de estimulación temprana no debe interferir con la vida emocional, educativa y social del niño. Es un deber de los gobiernos, asegurar la mejor calidad de vida de su población infantil, porque significa la mejor garantía para el desarrollo del país.

Los beneficios de la estimulación temprana son:

- Favorece el contactofísico y la compenetración adulta - niño.

- Permite al adulto descubrir las capacidades e intereses del niño. 
- Ayuda a construir la inteligencia en una etapa neurológica clave como es la de los primeros 5 años de vida del niño o niña.

- Dinamiza la personalidad, contribuyendo a que el niño se sienta satisfecho y eleve su autoestima al descubrir el alcance de sus potencialidades.

- Organiza una serie de actividades que permiten desarrollar las áreasmotriz, cognitiva y afectivo-social.

\section{Metodología.}

La investigación relacionada a "La estimulación temprana y el desarrollo psicomotor de los niños/as de 4 a 5 años del Centro de Educación Inicial "Eloy Alfaro" del cantón Riobamba, provincia de Chimborazo, en el año lectivo 2015-2016“, es no experimental, porque se realizó sin manipular deliberadamente las variables, es decir que se observó el fenómeno tal y como se da en su contexto natural, para después analizarlo y a partir de ello se procedió a la aplicación de técnicas y procedimientos de estimulación temprana que contribuyan al desarrollo psicomotor.

La investigación fue de tipo correlacional, porque midió el grado de relación existente entre dos o más conceptos o variables, en un contexto en particular, se realizó la relación entre dos variables, la correcta aplicación de técnicas de estimulación temprana y el adecuado desarrollo psicomotor en los niños y niñas de 4 a 5 años del Centro de Educación Inicial "Eloy Alfaro" de la ciudad de Riobamba, tuvo carácter bibliográfico porque se recopiló la información de libros, folletos, revistas, investigaciones científicas, las mismas que contribuyeron a determinar variables que fortalezcan el análisis, permitiendo tener una visión más amplia sobre el plan de estudio, también se le consideró de campo, porque se basó en informaciones obtenidas directamente donde se produjeron los hechos y fenómenos en el lugar de los acontecimientos, es decir en el Centro de Educación Inicial "Eloy Alfaro" de la parroquia Lizarzaburu, cantón Riobamba, provincia Chimborazo.

Se empleó el método hipotético-deductivo puesto que facilitó el proceso investigativo, que partió del enunciado del problema percibido a través de la observación de la realidad del mismo, se fundamentó de un marco teórico y planteamiento de la hipótesis para posteriormente elaborar conclusiones y recomendaciones.

Las técnicas e instrumentos utilizados se detallan a continuación:

La observación es elemento fundamental de todo proceso investigativo; en esta técnica nos apoyamos para indagar atentamente el fenómeno, tomar información y registrarla previo a su análisis obtener el mayor número de datos, para lo cual se utilizó una ficha de observación que permitió valorar la incidencia de la aplicación de técnicas de estimulación temprana y cómo éstas contribuyen en el desarrollo psicomotriz de los niños y niñas de 4 a 5 años. 


\section{Población y muestra.}

La presente investigación tiene una población de 25 estudiantes y 10 maestros tomando la totalidad de la muestra.

Cuadro No.1: Población y Muestra.

\begin{tabular}{lcc}
\hline \multicolumn{1}{c}{ Estratos } & Frecuencia & Porcentaje \\
\hline Estudiantes & 25 & 71.43 \\
Maestro & 10 & 28.57 \\
TOTAL & $\mathbf{3 5}$ & $\mathbf{1 0 0}$ \\
\hline
\end{tabular}

Fuente: Centro de Educación Inicial "Eloy Alfaro".

Elaborado por: Grupo de Investigadores.

\section{Procedimiento para el análisis e interpretación de resultados}

Una vez receptadas las fichas de observación, se procedió a la tabulación pregunta por pregunta, determinando sus frecuencias simples para luego transformarlas en porcentajes ubicados en cuadros estadísticos.

\section{Hipótesis General.}

La correcta elaboración y aplicación de técnicas de estimulación temprana, permite el desarrollo psicomotriz en las niñas y niños de 4 a 5 años del Centro de Educación Inicial "Eloy Alfaro", en la Parroquia Lizarzaburu, Cantón Riobamba, Provincia de Chimborazo. Período lectivo $2014-2015$.

\section{Hipótesis Específicas.}

- La investigación, analizó y aplico técnicas de estimulación temprana a través de juegos motores permiten el desarrollo psicomotriz en las niñas y niños de 4 a 5 años del Centro de Educación Inicial "Eloy Alfaro", en la Parroquia Lizarzaburu, Cantón Riobamba, Provincia de Chimborazo. Período lectivo 2015 - 2016.

- Las técnicas grafo plásticas permiten el desarrollo psicomotriz en las niñas y niños de 4 a 5 años del Centro de Educación Inicial "Eloy Alfaro", en la Parroquia Lizarzaburu, Cantón Riobamba, Provincia de Chimborazo. Período lectivo 2015 - 2016.

- Las técnicas pedagógicas (cognitivas) permiten el desarrollo psicomotriz en las niñas y niños de 4 a 5 años del Centro de Educación Inicial "Eloy Alfaro", en la Parroquia Lizarzaburu, Cantón Riobamba, Provincia de Chimborazo. Período lectivo 2015 - 2016.

\section{Resultados.}

Una vez receptadas las encuestas, se tabularon las preguntas, determinando sus frecuencias simples para transformarlas en porcentajes, incorporándoles sistema computable, para 
ubicarlos en cuadros estadísticos; se utilizó la estadística descriptiva utilizando el sistema porcentual, que sirvió para verificar la hipótesis, el cumplimiento de los objetivos, para finalmente justificar las conclusiones.

\section{1.- ¿Realiza actividades vinculadas con la estimulación temprana en su jornada diaria de trabajo?.}

Cuadro No.2: Pregunta 1.

\begin{tabular}{lc|r}
\hline \multirow{2}{*}{ Escala de evaluación } & \multicolumn{2}{c}{ Aplicación } \\
\cline { 2 - 3 } & Maestras & \multicolumn{1}{c}{$\%$} \\
\hline SI & 10 & $100 . \%$ \\
NO & 0 & 0.00 \\
TOTAL & 10 & $100 . \%$ \\
\hline
\end{tabular}

Fuente: Centro de Educación Inicial "Eloy Alfaro"

Elaborado por: Grupo de Investigadores

En la investigación la totalidad de las maestras aplican técnicas o estrategias de estimulación temprana en su jornada diaria de trabajo. Las maestras del Centro de Educación Inicial "Eloy Alfaro" con la ayuda de técnicas y práctica de estimulación buscan el desarrollo psicomotriz de los niños.

2.- ¿Qué actividades se realizan para la estimulación de los niños? .

Cuadro No. 3: Pregunta 2.

\begin{tabular}{lc|r}
\hline \multirow{2}{*}{ Escala de evaluación } & \multicolumn{2}{c}{ Aplicación } \\
\cline { 2 - 3 } & Maestras & $\%$ \\
\hline Presentación de videos para mover el cuerpo. & 1 & 10.00 \\
Juegos & 7 & 70.00 \\
Ejercicios grafo plásticos & 1 & 10.00 \\
Arte & 1 & 10.00 \\
TOTAL & 10 & 100.00 \\
\hline
\end{tabular}

Fuente: Centro de Educación Inicial "Eloy Alfaro".

Elaborado por: Investigadores.

El juego como estrategia de estimulación, el mismo que fortalece el cuerpo, la autoestima y despeja la mente de los niños permitiendo liberarse de sus temores y vincularse de mejor manera en la sociedad es utilizado por siete maestros, tres maestros motiva a sus niños a través de videos para mover el cuerpo, utiliza técnicas o estrategias grafo plásticas, aplica 
técnicas o estrategias de arte. Existen otras técnicas de estimulación como el arte y la pintura que buscan un objetivo común que es el desarrollo psicomotriz de los niños y niñas.

\section{3 ¿Con qué finalidad realiza actividades de estimulación temprana en los niños y niñas} de 4 a 5 años?

Cuadro No. 4: Pregunta 3.

\begin{tabular}{lc|r}
\hline \multirow{2}{*}{ Escala de evaluación } & \multicolumn{2}{c}{ Aplicación } \\
\cline { 2 - 3 } & Maestras & \% \\
\hline Mejorar la motricidad & 5 & 50.00 \\
Ayudar al desarrollo biopsicosocial del niño & 1 & 10.00 \\
Mejorar la coordinación viso - motora. & 0 & 0.00 \\
Estimular el aprendizaje & 3 & 30.00 \\
Desarrollar potencialidades & 0 & 0.00 \\
Desarrollar destrezas. & 1 & 10.00 \\
TOTAL & 10 & 100.00 \\
\hline
\end{tabular}

Fuente: Centro de Educación Inicial "Eloy Alfaro".

Elaborado por: Investigadores.

El ocho maestro buscan mejora la motricidad y el aprendizaje de los niños por medio de la estimulación temprana, dos maestros consideran que se puede conseguir el desarrollo social y el desarrollo de destrezas con sus niños.

4.- ¿Cuáles considera usted que son los beneficios prioritarios de la estimulacióntemprana en los niños y niñas de 4 a 5 años?.

Cuadro No.5: Pregunta 4.

\begin{tabular}{|c|c|c|}
\hline \multirow{2}{*}{ Escala de evaluación } & \multicolumn{2}{|c|}{ Aplicación } \\
\hline & Maestras & $\%$ \\
\hline Desarrollar y potenciar las funciones cerebrales de los niños y niñas & 5 & 50.00 \\
\hline Desarrollar el área cognitiva, social y motriz & 4 & 40.00 \\
\hline Desarrollo del Lenguaje & 0 & 0.00 \\
\hline $\begin{array}{l}\text { Reforzar aspectos intelectuales, físicos, sensoriales y sociales del } \\
\text { desarrollo }\end{array}$ & 1 & 10.00 \\
\hline Estrechar la relación adulto-niño & 0 & 0.00 \\
\hline TOTAL & 10 & 100.00 \\
\hline
\end{tabular}

Fuente: Centro de Educación Inicial "Eloy Alfaro".

Elaborado por: Investigadores. 
En el Centro de Educación Inicial "Eloy Alfaro" el noventa por ciento de los maestros consideran una prioridad el desarrollar y potenciar las funciones cerebrales de los niños y niñas, así como también el área cognitiva, y el diez por ciento equivalentes a un maestro considera que es importante el desarrollo social y motriz sin dejar de lado los aspectos intelectuales, físicos, sensoriales a esta edad.

5.- ¿Considera usted que la estimulación temprana incide en el aprendizaje de los niños y niñas de Educación Básica Inicial?.

Cuadro No.6: Pregunta 5.

\begin{tabular}{lcc}
\hline \multirow{2}{*}{ Escala de evaluación } & \multicolumn{2}{c}{ Aplicación } \\
\cline { 2 - 3 } & Maestras & \% \\
\hline Si & 10 & 100.00 \\
No & 0 & 0.00 \\
Total & 10 & 100.00 \\
\hline
\end{tabular}

Fuente: Centro de Educación Inicial "Eloy Alfaro".

Elaborado por: Investigadores.

La estimulación temprana incide en el aprendizaje y el desarrollo de los niños y niñas, la labor del docente fortalece la confianza y desarrollo de los niños, con una adecuada aplicación de técnicas y métodos de estimulación temprana acorde a su edad incidirá totalmente en los procesos de aprendizaje consideran todos los docentes.

6.- Que sabe Ud. de la importancia de conocer sobre el desarrollo de un niño desde que nace hasta la juventud.

Cuadro no.7: pregunta 6.

\begin{tabular}{lc|c}
\hline \multirow{2}{*}{ Escala de evaluación } & \multicolumn{2}{c}{ Aplicación } \\
\cline { 2 - 3 } & Maestras & \multicolumn{1}{c}{$\%$} \\
\hline Mucho & 3 & 30.00 \\
Más o menos & 6 & 60.00 \\
Poco & 1 & 10.00 \\
Total & 10 & 100.00 \\
\hline
\end{tabular}

Fuente: Centro de Educación Inicial "Eloy Alfaro".

Elaborado por: Investigadores.

Los maestros del Centro de Educación Inicial "Eloy Alfaro" dentro de su interés por conocer a los niños desde su nacimiento manifiestan tener un conocimiento modesto. En su totalidad 
los maestros tienen un conocimiento en esta área de estudio por lo que solo tres maestras están seguras de conocer sobre el paso de la niñez a la juventud, seis maestras conocen más o menos sobre el tema y un maestro tiene poco conocimiento del tema en estudio. Consideran importante investigar sobre el proceso que se da en el desarrollo de un niño desde que nace, crece, se forma un niño y posteriormente llegará a la juventud, teniendo muy en cuenta que las motivaciones que se le dé se reflejarán en su madurez.

\section{7.- ¿Qué sabe Ud. del desarrollo psicomotor en niños de 4 a 5 años?}

\section{Cuadro no.8: Pregunta 7.}

\begin{tabular}{lcc}
\hline \multirow{2}{*}{ Escala de evaluación } & \multicolumn{2}{c}{ Aplicación } \\
\cline { 2 - 3 } \multicolumn{1}{c}{ Maestras } & \% \\
\hline Mucho & 9 & 90.00 \\
Más o menos & 1 & 10.00 \\
Poco & 0 & 0.00 \\
Total & $\mathbf{1 0}$ & $\mathbf{1 0 0 . 0 0}$ \\
\hline
\end{tabular}

Fuente: Centro de Educación Inicial "Eloy Alfaro".

Elaborado por: Investigadores.

Las maestras del Centro de Educación Inicial "Eloy Alfaro" dentro de su amplio conocimiento y gracias a su práctica en un noventa por ciento son muy aceptable conocen las formas, métodos y técnicas cómo desarrollar a un niño tanto en lo intelectual, físico, social y psicológico, demostrando la importancia que tiene el desarrollo psicomotor en el ser humano desde sus primeros años, en la infancia y en la juventud, y un docente conoce el tema por su experiencia laboral.

8.- ¿En dónde cree que tienen origen los modos de conducta de un niño o niña de 4 a 5 años?

Cuadro No.9: Pregunta 8.

\begin{tabular}{lc|r}
\hline \multirow{2}{*}{ Escala de evaluación } & \multicolumn{2}{c}{ Aplicación } \\
\cline { 2 - 3 } & Maestras & \multicolumn{1}{c}{$\boldsymbol{\%}$} \\
\hline Se origina en el cerebro y en los sistemas sensorial y motor & 9 & 90.00 \\
Tiene su origen el sistema nervioso & 1 & 10.00 \\
Nunca se origina & 0 & 0.00 \\
Total & $\mathbf{1 0}$ & $\mathbf{1 0 0 . 0 0}$ \\
\hline
\end{tabular}

Fuente: Centro de Educación Inicial "Eloy Alfaro"

Elaborado por: Investigadores 
Solo un docente considera que el desarrollo de un niño depende de su sistema nervioso, y el noventa por ciento de los docentes conocen cómo desarrollar la psicomotricidad de un niño, cómo éste los va adquiriendo y cuál será su resultado final y como es lógico también manifiestan que el desarrollo del individuo depende mucho de su sistema nervioso central.

9.- ¿Qué aspectos considera usted que son importantes para que un niño de 4 a 5 años desarrolle su psicomotricidad correctamente?

Cuadro No.10: Pregunta 9.

\begin{tabular}{lc|r}
\hline \multirow{2}{*}{ Escala de evaluación } & \multicolumn{2}{c}{ Aplicación } \\
\cline { 2 - 3 } & Maestras & \% \\
\hline Dinámicas en la enseñanza & 2 & 20.00 \\
Medio ambiente adecuado en el sostén de desarrollo & 3 & 30.00 \\
Atmósfera familiar de sostén & 1 & 10.00 \\
Ayuda de un profesional idóneo & 3 & 30.00 \\
Alimentación adecuada & 1 & 10.00 \\
TOTAL & $\mathbf{1 0}$ & $\mathbf{1 0 0 . 0 0}$ \\
\hline
\end{tabular}

Fuente: Centro de Educación Inicial "Eloy Alfaro".

Elaborado por: Investigadores.

Las maestras consideran que el ambiente de trabajo y la ayuda profesional juegan un papel importante en el desarrollo del niño en sus primeros años, sin dejar de lado la ayuda familiar y un adecuado sistema de alimentación. Siendo un papel importante la dinámica en la enseñanza como un elemento para el desarrollo de la psicomotricidad .

\section{0.- ¿Cómo influye el Centro Inicial en el desarrollo psicomotor del niño de 4 a 5} años?

Cuadro No 11: Pregunta 10.

\begin{tabular}{lcc}
\hline \multirow{2}{*}{ Escala de evaluación } & \multicolumn{2}{c}{ Aplicación } \\
\cline { 2 - 3 } & Maestras & $\mathbf{\%}$ \\
\hline Porque adquiere habilidades motoras, gruesas y finas & 10 & 100.00 \\
Porque le ponen un examen y aprende & 0 & 0.00 \\
No aprende & 0 & 0.00 \\
Total & $\mathbf{1 0}$ & $\mathbf{1 0 0 . 0 0}$ \\
\hline
\end{tabular}

Fuente: Centro de Educación Inicial "Eloy Alfaro".

Elaborado por: Investigadores. 
El cien por ciento de los docentes concuerdan que durante la infancia se adquiere habilidades y destrezas motoras gruesas y finas es parte de la psicomotricidad infantil que son las acciones de habilidades motoras que implican el movimiento de los músculos en el cuerpo, la motricidad fina requiere movimientos muy precisos y coordinados.

\section{Conclusiones.}

- Un adecuado sistema de estimulación temprana influye en el desarrollo psicomotor de los niños y niñas de 4 a 5 años, del Centro de Educación Inicial "Eloy Alfaro" del Cantón Riobamba, Provincia de Chimborazo, en el año lectivo 2015-2016 y por lo tanto contribuye al mejoramiento de su calidad de vida.

- La investigación, analizo y aplicó técnicas de estimulación temprana a través de juegos motores gruesos permiten el desarrollo psicomotriz en las niñas y niños de 4 a 5 años del Centro de Educación Inicial "Eloy Alfaro", en la Parroquia Lizarzaburu, Cantón Riobamba, Provincia de Chimborazo. Período lectivo 2015 - 2016.

- La técnicas pedagógicas (cognitivas) permiten el desarrollo psicomotriz en las niñas y niños de 4 a 5 años del Centro de Educación Inicial "Eloy Alfaro", en la Parroquia Lizarzaburu, Cantón Riobamba, Provincia de Chimborazo. Período lectivo 2015 2016.

\section{Referencias bibliográficas.}

- Arango, L. (2007). Estimulación Temprana. Estimulaatubebe.blogspot.com. Retrieved 18 February 2016, from http://estimulaatubebe.blogspot.com/

- Arce, C. (2015). Desarrollo Psicomotor. Arcesw.com. Retrieved 18 February 2016, from http://www.arcesw.com/dpm.htm

- Author stream, (2013). Estimulación Temprana - Fundamentos Psicopedagógicos. Author STREAM. Retrieved 18 February 2016, from http://www.authorstream.com/Presentation/RodnyAC-1566791-estimulaciontemprana-fundamentos-psicopedagogicos/

- CARRANZA, C., LEÓN, K., \& NEGREROS, S. (2012). Estimulación Temprana Monografía. Scribd. Retrieved 18 February 2016, from http://1948f70508a693849b622441e0d31b1f.proxysheep.com/doc/136987979/Esti mulacion-Temprana-Monografia Cosas de la Infancia, (2013). DESARROLLO PSICOMOTOR DEL NIÑO DE 4 A 5 AÑS. Cosasdelainfancia.com. Retrieved 18 February 2016, from http://www.cosasdelainfancia.com/biblioteca-nino09.htm 


\section{Para citar el artículo indexado.}

Barrera H., Flor A., \& Flor F. (2018). Estimulación temprana y desarrollo psicomotor en niños 4 a 5 años. Revista electrónica Ciencia Digital 2(1), 60-73. Recuperado desde: http://www.cienciadigital.org/revistascienciadigital/index.php/CienciaDigital/article/view/5 15

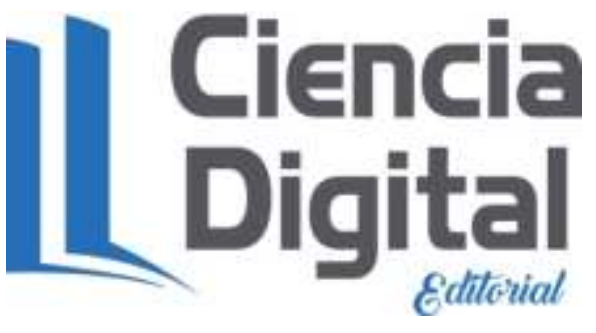

El artículo que se publica es de exclusiva responsabilidad de los autores y no necesariamente reflejan el pensamiento de la Revista Ciencia Digital.

El articulo queda en propiedad de la revista y, por tanto, su publicación parcial y/o total en otro medio tiene que ser autorizado por el director de la Revista Ciencia Digital.
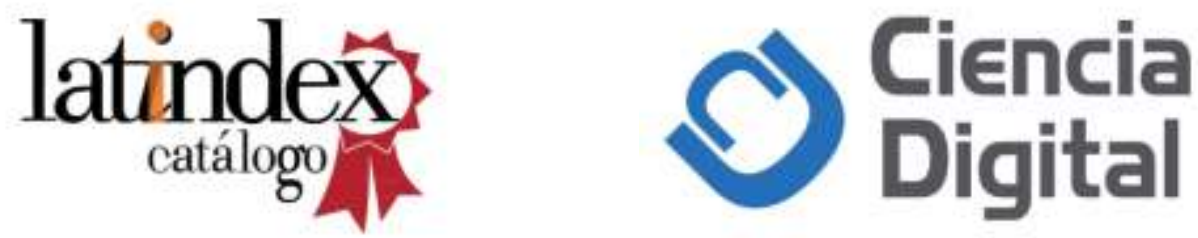